\title{
Spatial Data Infrastructure Requirements for Mobile Location Based Journey Planning
}

\author{
Jessica Smith \\ Department of Geomatics \\ University of Melbourne \\ Allison Kealy \\ Department of Geomatics \\ University of Melbourne
}

\author{
William Mackaness \\ Department of Geography \\ University of Edinburgh \\ Ian Williamson \\ Department of Geomatics \\ University of Melbourne
}

\begin{abstract}
A growing number of services are now being offered over mobile devices. They typically combine positioning technology, wireless technology and spatial analysis methods applied to detailed geographical, time based data to offer services in support of time critical, spatial, mobile decision making. A collection of research issues need to be addressed in the successful delivery of such services that extend beyond issues of sophisticated network algorithms. Specifically, careful attention needs to be given to: (1) people and user environments; (2) access to networks; (3) policy, privacy and liability; (4) standards and interoperability; and (5) data quality. Spatial Data Infrastructure (SDI) is the collective term for these interconnected issues and has been a traditional area of research associated with geographic information science. In this paper the particular SDI requirements for the successful delivery of Location Based Services (LBS) are explored through the development of a prototype LBS for journey planning. The initial implementation and testing of this prototype has revealed that the SDI context is well suited as a framework within which to examine the related LBS issues. From a more technical perspective, the testing has revealed that data structure and the means by which large data sets are mined (in order to gather information to present to users) is critical to the success of timely information delivery over limited bandwidth media.
\end{abstract}

Address for correspondence: Ian Williamson, Department of Geomatics, The University of Melbourne, Victoria 3010 Australia. E-mail: ianpw@unimelb.edu.au 


\section{Problem statement}

The proliferation of mobile phones into society has led to a changing communication paradigm, described by some as a paradigm shift in how information is accessed and utilised in a 'mobile centric' world (Sacher and Loudon 2002). It is certainly the case that mobile phones have provided people with an unparalleled freedom to communicate in a variety of modes irrespective of their location (Singleton 1983, Cox 1996, Solymar 1999). Many mobile phones are Java enabled and support the Wireless Application Protocol (WAP) via GSM (Global System for Mobile Communication) or GPRS (General Packet Radio Service). Mobile phone functionality is also starting to be integrated with personal digital assistants (PDAs) and presence technologies (such as the Global Positioning System (GPS) and Bluetooth) (Blonz and McCarthy 1998, Pratt 1999, Koh and Kim 2000) forming one device capable of recording and organising tasks and communicating with others. Predictions in the level of adoption, and the type of services offered abound (refer to Blonz and McCarthy (1998), McCabe (1999), Robinson (2000), Souissi and Phillips (2000), and Dennis (2001) for additional details). Ericsson $(2000,2001)$ predict that there will be more mobile internet subscribers than fixed lines by 2003 , with the number of mobile phones in circulation now exceeding 1 billion world-wide. Current and proposed services range from business support through to entertainment (Figure 1) and collectively the technology is fundamentally changing how we manage our day to day activities (Carroll et al. 1991).

A subset of the services listed in Figure 1 involve the use of geographical information; information that is current and intended for immediate use as part of the decision making process. For example someone in a car may wish to detour a traffic accident; a tourist may wish to know if there is sufficient time to visit a gallery, together with the most appropriate mode of transport and a navigable route description to guide them there. Where the decision making process involves the use of a service based on geographical, timely data with respect to the position of the decision maker, the service can be defined as a location based service (LBS). Encompassing 'pull' services such as journey planning (including navigation and route modification facilities), optimal scheduling and execution of a set of activities, and 'push' services (such as proximity based advertising), LBS require careful consideration of issues such as network loads, privacy, standards (central to the idea of interoperability), and the associated policy issues. It is also necessary to resolve problems in the integration and management of fine scale, spatial and non-spatial data, and how it is gathered and presented to the user. Within the spatial information science discipline, these issues have traditionally been explored within the framework of the Spatial Data Infrastructure (SDI) - a concept that has been evolving over the past decade. Thus, the central questions are: How will the proliferation of LBS impact SDI? How should existing ideas on SDI be extended to comprehensively accommodate the successful delivery of such services?

This paper begins to address these questions in the reverse order in which they are posed. In addition to a theoretical discussion, this paper identifies how the current SDI requirements need to adapt to support LBS (the second question) through the implementation of a public transport location based service for Melbourne, Australia. An outline of the phased prototype implementation is provided, along with a discussion of how the implementation is beginning to identify modifications required in SDI modelling. In turn, these findings are expected to help examine how the proliferation of LBS will impact on SDI (the first question), and as such will form the basis for a subsequent 
Email Messages

Driving Directions

Traffic and Driving Updates

Weather Information

Telephone Directory Search

Personal or Business Reminders

Local Services Information

Personal Banking Services

Stock Quotes, Market Information

General News Updates

Entertainment Directories

Theatre/Movie Ticket Ordering

Latest Sports Scores

None of the above

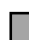

$49 \%$

Figure 1 Mobile Internet application preference (Bourrie 2000)

paper. It is highly likely in the short term that LBS will continue to be developed by private industry with little regard for bigger picture infrastructure ideals. On the other hand, some government initiatives are more forward thinking and are already including LBS in their spatial information strategic planning dialogue. As a result, the infrastructure required to deliver such services is being re-examined.

\section{A Definition of LBS}

The delivery of services over mobile devices is a huge growth industry. With mobile phones expected to outnumber fixed line phones in 2002 (Ericsson 2000), the provision of position sensitive, time critical information to mobile users through a variety of devices is an emerging area, encouraged by legislative mandates in the USA and similar initiatives in Europe (refer to FCC (1999) and Parthus Technologies (2001), respectively for additional details). A location based service is concerned with supporting dynamic spatial decision making through the provision of real-time, geographically based information (typically at a fine scale).

As described earlier, this decision making process is from the perspective of an individual (rather than a group or organisation etc.) concerned with coming to a decision 
about getting from one place to another. The decision making process could involve deliberating over the means of transportation or the transportation route itself. Within this context, spatial decision making is about coming to a decision that is related (in either the procedure or the final result) to a geographical or spatial entity (for example a place or location). The dynamic component relates to the changing environment in which the individual is operating. This change can be a function of time, the geographical position of the individual, or the tasks that the user is performing. For example, if en route from $A$ to $B$ a traveller decides to stop at a point $C$, they should be able to use the LBS to alter their current route, to include the new change. Similarly, if being guided by navigation directions from $\mathrm{A}$ to $\mathrm{B}$ and a wrong turn is made, they should be able to use the LBS to obtain new directions (ideally the system should automatically detect and deal with this case). The flexible and adaptive decision making process of individuals (Payne et al. 1993), in conjunction with an often changing and complex task environment demands significant, often tailored support.

From a business perspective, the user must have a clear understanding of the value in utilising a specific location based service. Typical LBS applications require fine scale data and utilise spatial analysis techniques that support the presentation of information in a variety of forms (not merely two dimensional maps). The constraints of interface require careful structured presentation of the data, often in a linked, hierarchical form (Hoogenraad 2000, Quirion 2001). In line with Tobler's first law, the relative importance of geographical information is invariably governed by its nearness to the user (in both time and space); thus the integration of positional technology is central to any LBS device.

In summary, Location Based Services are a result of the convergence of position, information and communication technologies as shown in Figure 2, and encompass issues of human computer interaction (modes of interaction and immersion, decision making, activity scheduling and situational awareness), data (metadata, standards, scale, multi-modal delivery and dynamic updates), spatial analysis (topological modelling, information trawling, integration and conflation, and abstraction and generalisation), people (personalisation, computer literacy, spatial literacy, social or cultural influences) and applications (revenue models, policy, privacy, role of value added resellers). Whilst each of these individually is worthy of pursuit, the philosophical underpinning of this

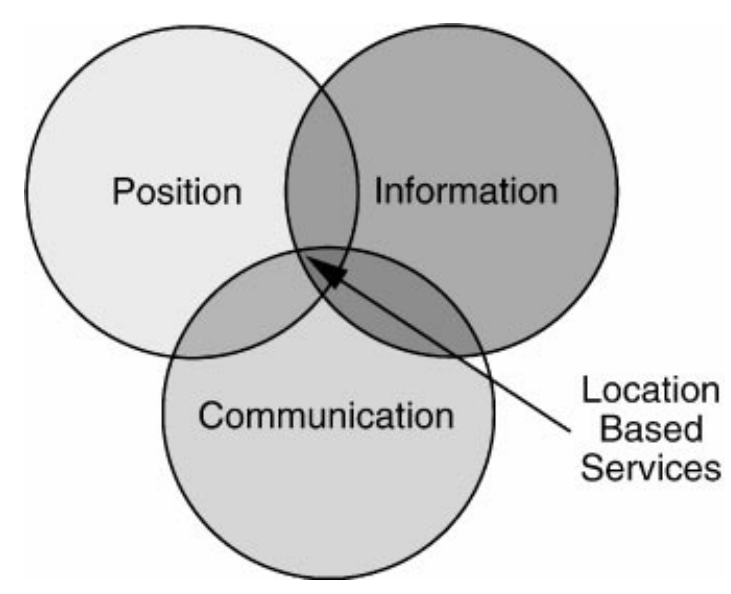

Figure 2 Location Based Services: The convergence of technologies 
research relies on the relationship and interdependencies between each of these components. For example, planning a multi modal journey requires detailed information from the user (an interactive environment to support this process); detailed, current schedules for a range of transport networks and transfer points between modes; analysis methods for deriving a solution; and text, image and sound techniques for communicating that solution.

To investigate these interdependencies, this research focuses on the development of an LBS that will enable users to plan and execute routes using the public transport system in the city of Melbourne. Through user needs analysis it is possible to define the requirements of such a system: operating on a device that can be personalised, the application will respond to the location of the user, relay public transport vehicle positions within the network in a dynamic fashion, record routes for future use with the aim of conveying this information efficiently, in a memorable form, consistently and responsively. The information needs to be verifiable such that the user can perform the two core tasks of planning and navigating in addition to other tasks associated with their mobility. More specifically the mode of interaction needs to support activities such as:

1. Allowing definition of (multiple) start, way and end points;

2. Enabling lookup facilities of past plans (facilitating personalisation);

3. Displaying route information in a variety of forms, and in varying detail (thus providing context);

4. Providing guidance and confirmation information prior to, and during the journey;

5. Providing updates and consideration of alternate proposals based on changing conditions of the network, and of the user's activity scheduling; and

6. Supporting estimation of time and distance.

Before looking at these issues in more detail, a review of the essential elements of a current SDI model is provided, and the link between SDI and LBS is explored further.

\section{Spatial Data Infrastructure and Location Based Services}

The increased use and importance placed on spatial information across many discipline areas, and the associated need for cooperation between spatial data users and producers has led to a recognition of the need for an infrastructure for land and geographic information, a Spatial Data Infrastructure (SDI). SDI are intended to create an environment which enables a variety of users to access and retrieve complete and consistent data sets in an easy and secure manner (Coleman and McLaughlin 1998, Rajabifard et al. 2000). Typically this environment is created through the development of dynamic partnerships between inter- and intra-jurisdictional organisations. However as Feeney and Williamson (2000, p. 95) indicate, SDI also 'have the potential to increase business opportunities for the geographic information industry, and promote widespread use of available data sets'. Encompassing issues of people and user environments; network access; policy, privacy and liability; standards and interoperability; and data quality, SDI enable efficient collection, management, access, delivery and utilisation of spatial data.

Relying on a range of integrated data sets (both spatial and non-spatial), LBS must extract relevant portions of these data sets and present them to users in an appropriate 


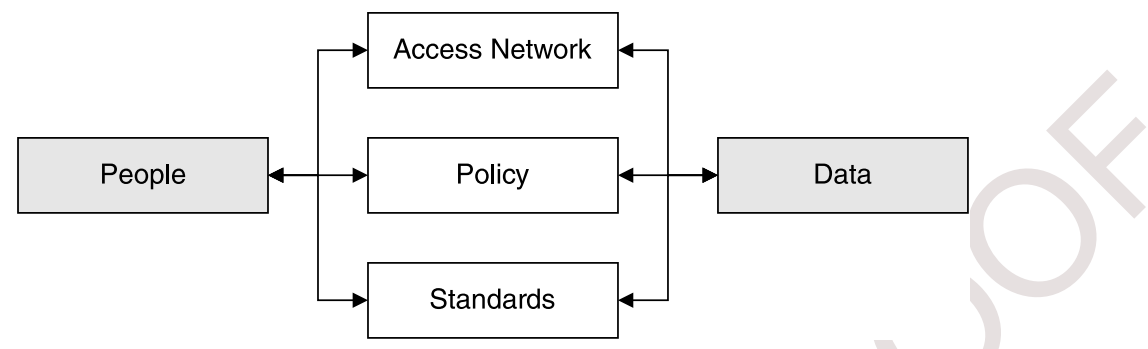

Figure 3 SDI components (Rajabifard et al. 2000)

form. In order to achieve this, appropriate policies for data use must be in place, and communication methods defined for wireless information transmission. These broad requirements parallel the five SDI components of people, access networks, policy, standards and data. From an LBS perspective, the essential elements are the people (encompassing the diversity of users and producers of spatial data, both accessing and as 'input' to the service), and the data necessary to support the service (particularly its currency, completeness and integration). For the data to become useful information it is necessary to have in place the networks, together with policies governing use, and access, and standards relating to the quality of data, and its integration. These ideas are summarised in Figure 3.

Each of these components is critical within an SDI, and strongly related to the others thus forming the cohesive nature of the infrastructure. Additionally through the recognition of these components in a range of SDI implementations, an integrated, hierarchical structure of SDI from corporate to global levels has been identified (Chan and Williamson 1999; Rajabifard et al. 1999, 2000). As a result, determining SDI requirements in the context of LBS forces an examination of all of the components, rather than focussing on any one individual component in isolation. Redefining one component will impact on others. For example, the policy component will have to examine privacy in response to the concerns of the community in relation to the ability of a mobile phone to determine its location, transmit and use this information to filter content. At the same time, the technical standards component will have to consider metadata and ways of ensuring the quality of the data provided to users.

As mentioned earlier, the original intention of SDI was to create an environment within which a variety of users could access and retrieve complete and consistent data sets easily and securely (Coleman and McLaughlin 1998). Unlike traditional SDI end users, LBS end users will not require access to complete data sets in the sense of coverage at small scale. They will, however, require detailed information relevant to their current location and surrounding area. Recently, the importance of the end user in the SDI setting has become more apparent. Unlike users of a state government SDI, the users of an LBS may not necessarily be trained in spatial analysis and interpretation. Vector and raster data sets must be 'translated' into a form that is easily digestible and memorable by the general public, and 'fits' within the restrictions of wireless communication and associated devices.

Although SDI models have proved sufficient for spatial analysis, long term planning and decision making, as well as other relatively static modelling purposes, mobile services place new requirements on the use and delivery of geographic information. This arises from the properties of LBS which can be described as being concerned with: 
Table 1 SDI requirements in light of LBS delivery

\begin{tabular}{ll}
\hline Component & Requirement \\
\hline Access: & Speed or scalability of service \\
& Ease of production of information \\
& Ease of consumption of information \\
& Methods of interaction and personalisation \\
& Knowledge of location of people and/or activities \\
Policy: & Correctness of information \\
& Critical nature of decision making \\
& Greater integration and interoperability \\
Standards: & Quality of data (currency, precision) \\
& Varying resolution and granularity of information \\
\hline
\end{tabular}

- information exchanges that are time and space critical;

- supporting dynamic spatial decision making (with the consequent need for immediacy and flexibility);

- information that is proximity critical (the closer you are to an activity, the more important it is to know about that activity);

- information display on a device of limited affordance and real estate; and

- the competition of tasks and events whose outcome may then influence subsequent decisions (such as changes in weather, tasks taking longer to complete than expected, etc.).

These issues relate to the 'user centred' dissemination of information, but they can also be linked to the technical SDI components as summarised in Table 1.

Thus the demands and requirements of the mobile nature of LBS end users are highlighting limits to the current SDI model. While the generic SDI principles of people, access, standards, policy and data are still of prime importance to LBS, more detailed definitions for each of these components is required to meet the challenges of the mobile, wirelessly connected end user.

\section{Methodology}

The purpose of this research is to use a set of phased implementations of a public transport information system to explore and extend the understanding of SDI requirements.

The strong user focus and the time critical nature of this service lends itself to Jacobson's method of object-oriented software engineering (OOSE) (Jacobson et al. 1992). The OOSE method involves the formation of models that capture the actors of the system and their behaviour for each of the design stages. As the method name suggests, the models are made up of objects representing real world entities. This is a natural way for people to describe their environment; therefore, the semantic gap between the models and the real world is relatively small.

Although not specifically intended for prototyping, following the OOSE approach in the prototyping process can prove useful to the final system (Jacobson et al. 1992). 


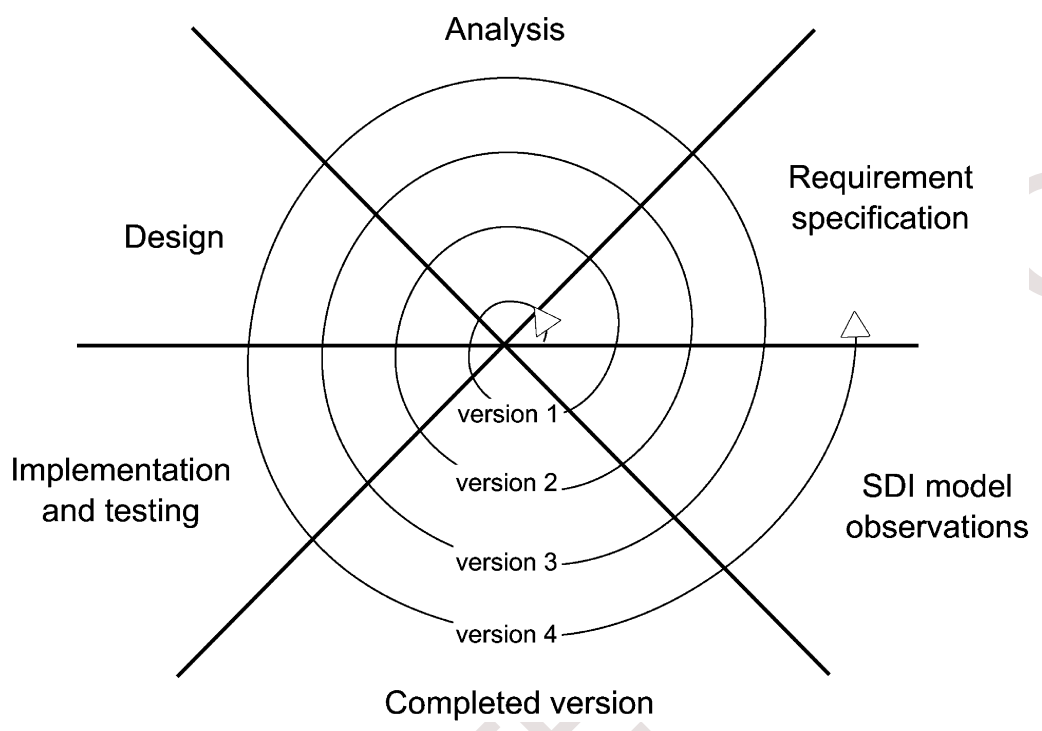

Figure 4 Components of design feeding through to implementation and observations of SDI requirements (adapted from Jacobson et al. 1992)

Table 2 Proposed prototype implementations

\begin{tabular}{ll}
\hline Version & Functionality/Description \\
\hline 1 & Tram route finder (from one tram stop to another) \\
2 & Tram route finder with pedestrian navigation to first stop, and from last stop \\
3 & (where necessary) \\
4 & Tram route finder with integration of real-time tram location information \\
& Public Transport route finder - trams and buses or trains - with pedestrian \\
& navigation and real-time updates as appropriate \\
\hline
\end{tabular}

The OOSE method of software development process involves a sequence of requirements specification, analysis, design, implementation and testing. As is noted by Henderson (1991) and Jacobson et al. (1992) this process is usually cyclic or incremental in nature following the development of the product - each implementation refines the analysis and design stages through evaluation and testing of a completed version (Figure 4). In the context of this research, this successive version development is anticipated to lead to a more informed view of SDI requirements.

The incremental development strategy allowed the problem of constructing a public transport information system to be tackled in smaller, more manageable portions of increasing complexity. In addition, it was expected that each version would reveal unique features related to the requirements of the underlying infrastructure and enable exploration of the interfaces between the SDI components. A summary of the prototype versions is given in Table 2, and the SDI features that were explored through the first version of the prototype are shown in Figure 5. 


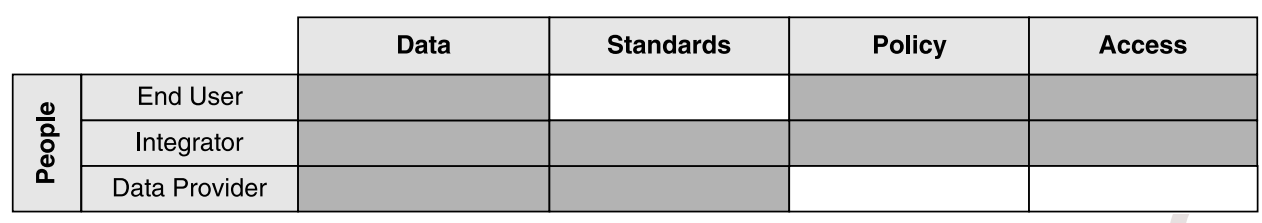

Figure 5 Dark grey boxes represent the SDI elements explored through version 1 of the prototype

The public transport information system can be classified as a guidance service. Guidance services encapsulate the dual tasks of planning a journey, and supporting navigation in 'following' a planned route. Both these tasks require delivery of potentially complex geographic information at a range of scales (from the broad scale of journey planning which requires a conceptual view of the entire region encompassing alternate transport mediums to the fine scale in portraying landmarks and cues in the immediate vicinity of the traveller - in supporting the process of situational awareness). Typically the user requires 'user centred' information rather than data covering a large geographical extent. Extracting the relevant portions of data sets and presenting them in an appropriate format is a challenge for LBS developers.

Public transport networks change spatially, topologically and temporally and systems are required that can cope with mobile, dynamic decision making (Costelloe et al. 2000), that accommodate the task of activity scheduling (Huisman and Forer 1998) and that take into account route length, travel mode, time, and frequency of mode changes. Peng and Huang (2000) define a taxonomy of transit information systems based on function and content. Using this model, information content can range from general, static information through to real-time information (including vehicle positions and delays). The function level and interface of transit information systems can span simple web browsing at the lowest level through to customised information delivery and online ticket transactions at the highest level. By assigning increasing integer values to each function level, and increasing alphabetical characters to each content level, Peng and Huang (2000) define a useful framework by which to classify transit information systems. The ultimate version of the public transport information system under development would be one that incorporates real-time content with customisation and information delivery (a D3 service according to Peng and Huang (2000)). For this project, a series of prototype versions will range from an application that uses static information with text searches and static graphic images (a B1 service (Peng and Huang 2000)), up to the D3 level.

\subsection{Requirement Specification and Analysis}

The requirements stage of the design aims to define the limitations of the system and specify the system's behaviour from the perspective of a user (Jacobson et al. 1992). The construction of use cases (based on discussions with members of the ISO/TC211 working groups on Location Based Services, public transport use scenarios developed by the authors and the work of Kjeldskov et al. (2003)) detailing how a user would interact with the system identified the various system objects (Figure 6) and highlighted the need for careful consideration to be given to issues of human computer interaction within the mobile environment. 


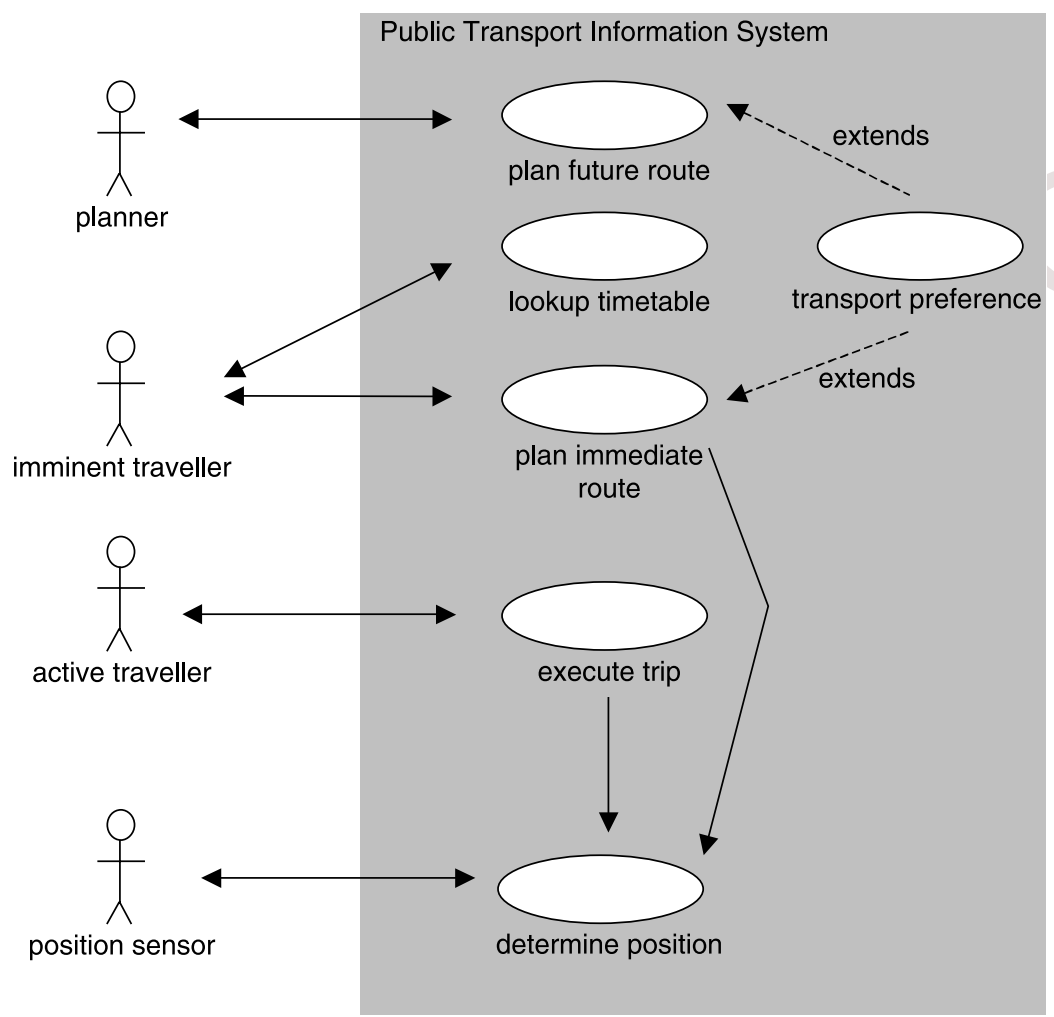

Figure 6 Prototype use case model

$\mathrm{s} 11 \mathrm{rt19}$ | s27
$\mathrm{x} 8 \mathrm{rt24}$ O s2

(a)

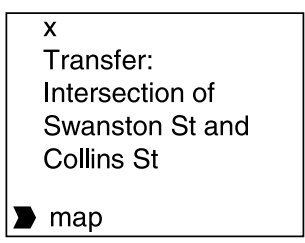

(b)

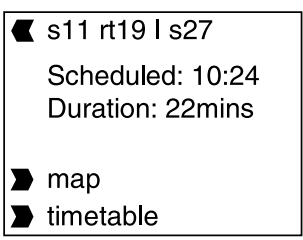

(c)

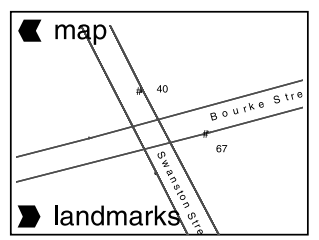

(d)

Figure 7 Proposed interface (a) raw text, (b) landmark descriptions, (c) route description, or (d) maps

A broad range of alternatives exist for the portrayal of route information, from simple, a-spatial text (Figure 7a), through 'geographical text' such as landmark information (Figure $7 \mathrm{~b}$ ), to graphical map based solutions (Figure $7 \mathrm{~d}$ ).

Text (such as in Figures $7 \mathrm{a}$ and $7 \mathrm{c}$ ) is abstract, readily remembered, and simple to interpret, and would suffice for a user familiar with the tram system. Description of the environment (Figure 7b) can be used to clarify waypoints along the journey. Information of the form shown in Figure $7 d$ would require the user to interpret and navigate (using skills in orientation and positioning). It is easy to envisage different environments 
Table 3 The heuristics of good design for mobile environment location based services (based on Molich and Nielsen (1990) and Nielsen (1994))

\begin{tabular}{|c|c|}
\hline Visibility: & $\begin{array}{l}\text { Visibility of system status (informing of what the system is } \\
\text { doing), alternative actions. }\end{array}$ \\
\hline Responsive: & Efficient in delivery and interpretation of information. \\
\hline Reinforcement: & $\begin{array}{l}\text { Confirmation of location (e.g. through landmark information) } \\
\text { increases confidence. }\end{array}$ \\
\hline Reversibility: & Ability to backtrack mistakes and re-enter data. \\
\hline Adaptability: & $\begin{array}{l}\text { Information presented in a variety of forms, customised to } \\
\text { user's geographical knowledge and needs and preferred mode } \\
\text { of interaction (e.g. keystroke/shortcuts or stylus). Includes } \\
\text { personalisation features. }\end{array}$ \\
\hline Predictability: & User control (or system) anticipates information requirements. \\
\hline Structure: & Organisation of data (e.g. according to task/level of detail). \\
\hline Consistency: & $\begin{array}{l}\text { Principle of least astonishment and coherence in the operation } \\
\text { of keys, menus, etc. }\end{array}$ \\
\hline Compatibility: & Follows from previous experience in using similar devices. \\
\hline Good mappings: & $\begin{array}{l}\text { It is possible to determine the relationships between actions } \\
\text { and results, between the controls and their effects, and } \\
\text { between the system state and what is visible. }\end{array}$ \\
\hline Multi-tasking: & Complimentary to other competing tasks. \\
\hline Economy: & Few steps (keystrokes) in reaching information. \\
\hline Memorability: & $\begin{array}{l}\text { Information readily assimilated or memorised to reduce revisits } \\
\text { to device. }\end{array}$ \\
\hline Abstraction: & Irrelevant information impairs short term recall. \\
\hline & System error trapping (e.g. through fixed choice menus). \\
\hline & $\begin{array}{l}\text { Support user in recognising/recovering from errors/missing } \\
\text { information. }\end{array}$ \\
\hline Help or Documentation: & $\begin{array}{l}\text { Providing information (on-line and as a manual) in the use of } \\
\text { the system. }\end{array}$ \\
\hline
\end{tabular}

in which each of these (or a combination of these) is most appropriate. Information in these forms lend themselves to a hierarchical structuring according to the level of detail and whether the user is planning or executing a journey (for example in the case of planning a journey, the hierarchy may move from a-spatial text, through landmark and route descriptions, and thence to maps).

Interface design and usability principles are a well researched and documented area, and while Nielsen and Molich's general heuristic principles (refer to Molich and Nielsen (1990) and Nielsen (1994) for additional details) are still relevant to the mobile environment, some revisions are required. A specific set of heuristics for wireless location based services is given in Table 3 .

In the context of this research and the examination of SDI, the interface design was of secondary importance. However it still focused around these heuristics and a hierarchical information structure from simple text descriptions through to two dimensional maps was employed; the graphical elements of the prototype were developed with the assistance of Webraska's spatial query and mapping tools. 


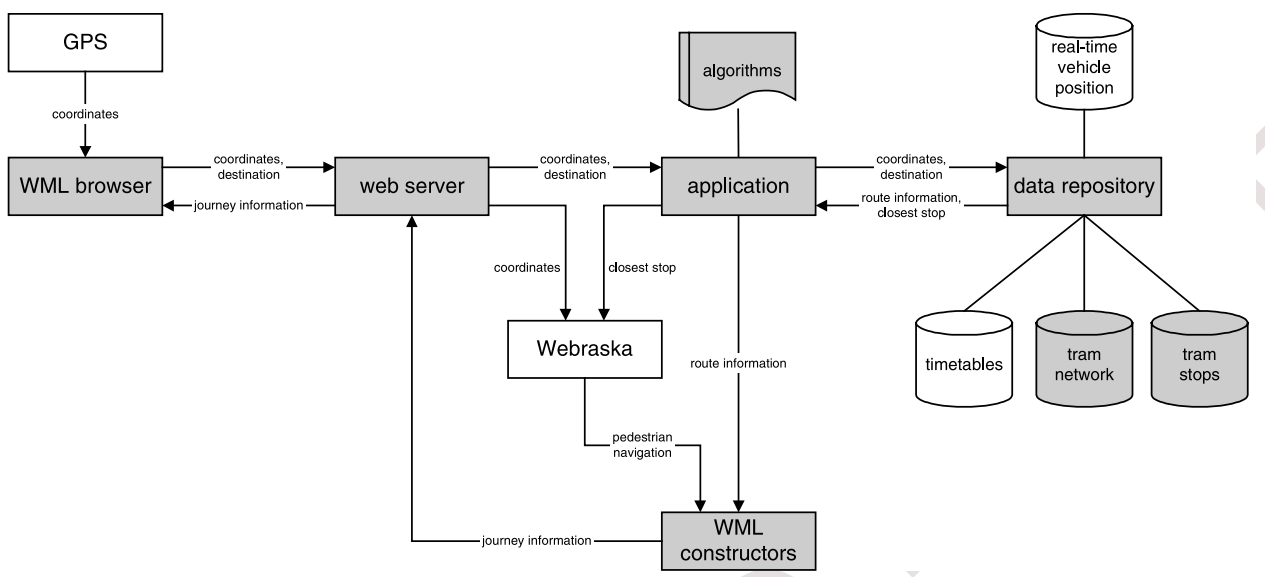

Figure 8 Prototype structure (version 1 components in grey)

\subsection{Implementation and Testing}

Achieving the desired user interface and functionality for the prototype application required the integration of several components. The structure of the final system is summarised in Figure 8. Even though only the first version has been implemented, the main structural components had to be determined from the outset. All but four components of the final configuration (GPS, Webraska, real-time vehicle positions and timetables) have been included in the first version of the prototype.

The user interface was implemented using the Wireless Markup Language (WML). The user interacts with the service via a WML browser on a mobile phone or Internet enabled PDA. Service requests are passed from the browser to the web server which redirects the public transport routing requests to the route finding application (and will pass the initial position of the user (obtained via GPS) to Webraska's service in preparation for pedestrian navigation guidance to the closest tram stop). The application communicates with the data repository to determine an appropriate route. The navigation information is combined and constructed into a hierarchical series of WML documents that are presented by the web server to the user's browser.

Examination of each of the SDI components and in particular expectations of what issues would be revealed through the construction and testing of the prototype led to the formulation of the observation matrix shown in Figure 9. Each version of the prototype is expected to reveal new issues or examine previously identified issues in more detail. For example, response time was explored in version 1, and will also be examined in subsequent versions where the amount and type of data presented to the user increases in richness.

The version one implementation was tested on an IPAQ pocket PC with a live GPRS wireless connection. Access issues were predominantly tested quantitatively, with the other issues requiring more subjective qualitative analysis. The quantitative testing process involved simulating use of the system by a user to find a route between two specific tram stops. The time taken for the user to access the service and request a route solution was recorded along with the amount of data transmitted wirelessly. This allowed the 'calculation' of representative costs for obtaining information (effort required to use the service and service charges) to be compared against the benefits (immediacy, convenience, completeness, currency). 


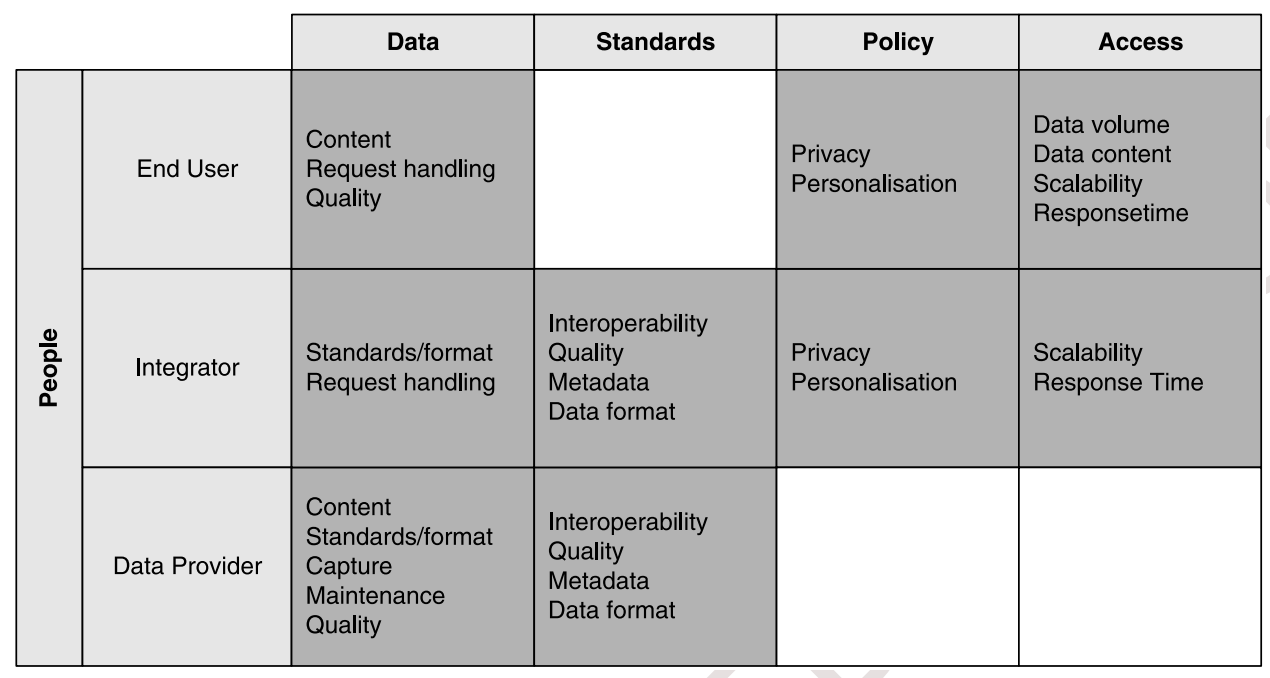

Figure 9 Observation matrix for version 1 of the prototype

Three sample routes were used in the testing process. The routes covered different scenarios and as a result returned varying quantities of information. Test route 1 was a simple journey of six stops with no interchanges. Test routes 2 and 3 both required interchanges from one tram route to another. The journey for route 2 required only one interchange (and passed through a series of six stops), while the route 3 journey required two interchanges and passed through ten stops. The testing process was repeated six times for each route to determine mean values and rule out anomalous results. Even though this is a relatively small testing sample, the results obtained were relatively consistent and contributed to meaningful analysis.

Some analysis was also performed on the web server to determine scalability capabilities. Access to the specific URLs that activate the route generation by the application were tested for speed and bandwidth use. Again this was conducted in a series of runs to obtain realistic, representative values.

\section{Results}

The three test routes demonstrated increasingly complex tram journeys, and hence the number of screens required to display the information to the user increased with each route (as is shown in Figure 10). Since route 1 was a simple journey that could be completed by boarding a single tram, only one result screen was presented. Routes 2 and 3 required the traveller to use two and three trams for their journey respectively, hence additional display screens were provided with information about these vehicle interchanges.

The following two tables show the times recorded to use the system and receive a response. Times were recorded for each of the six runs for each test route. Table 4 shows the breakdown of times for the transition between individual pages. Page 1 is the initial page that prompts the user for the tram stop numbers for their journey origin and destination; the other pages are numbered as per Figure 10. 


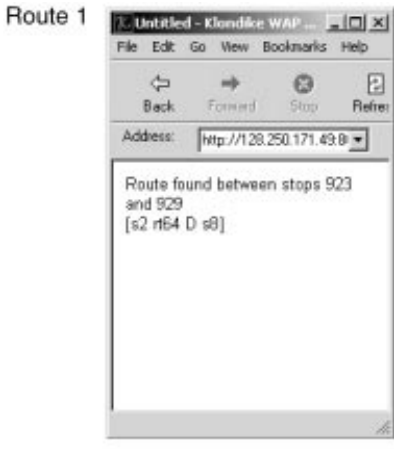

Page 2

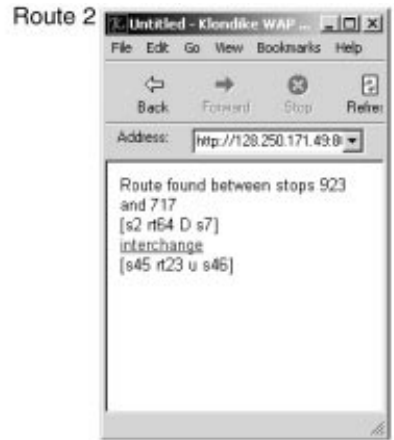

Page 2

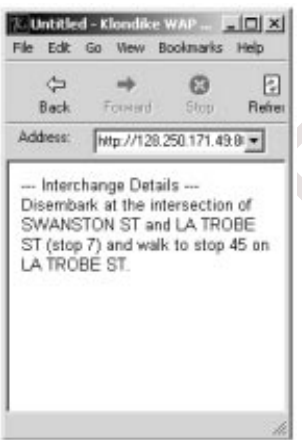

Page 3
Route 3

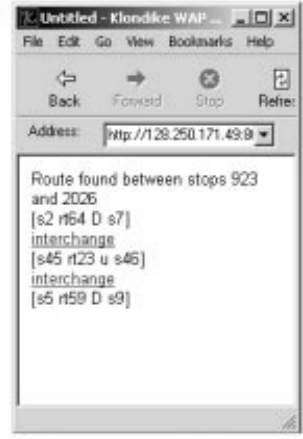

Page 2

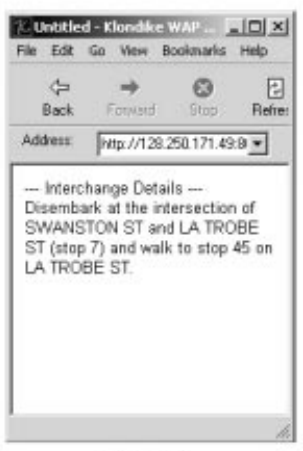

Page 3

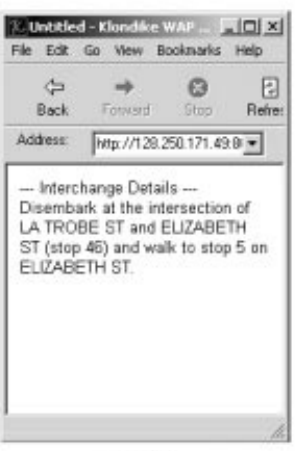

Page 4

Figure 10 Examples of information presented to the user for each of the test routes

Table 4 Application Use - Response time per page

\begin{tabular}{|c|c|c|c|c|c|c|}
\hline \multirow[b]{2}{*}{ Route } & \multicolumn{2}{|l|}{ Page } & \multicolumn{4}{|c|}{ Times (seconds) } \\
\hline & From & to & mean & $\min$ & $\max$ & $\sigma$ \\
\hline \multirow[t]{2}{*}{1} & 1 & 2 & 14 & 11 & 19 & 03 \\
\hline & 2 & 1 & 08 & 05 & 13 & 03 \\
\hline \multirow[t]{4}{*}{2} & 1 & 2 & 13 & 08 & 22 & 05 \\
\hline & 2 & 3 & 10 & 07 & 26 & 07 \\
\hline & 3 & 2 & 13 & 05 & 47 & 15 \\
\hline & 2 & 1 & 07 & 03 & 13 & 03 \\
\hline \multirow[t]{7}{*}{3} & 1 & 2 & 14 & 11 & 17 & 03 \\
\hline & 2 & 3 & 09 & 06 & 23 & 06 \\
\hline & 3 & 2 & 11 & 06 & 33 & 10 \\
\hline & 2 & 4 & 06 & 01 & 23 & 08 \\
\hline & 4 & 2 & 09 & 07 & 12 & 02 \\
\hline & 2 & 1 & 06 & 03 & 11 & 03 \\
\hline & Avera & & 10 & & & \\
\hline
\end{tabular}


Table 5 Application Use - Client Perspective

\begin{tabular}{lllllll}
\hline & \multicolumn{2}{l}{ User Time (seconds) } & & \multicolumn{2}{l}{ Data (bytes) } & \\
\cline { 2 - 3 } \cline { 5 - 6 } Route & mean & $\sigma$ & & mean & $\sigma$ & Cost \\
\hline 1 & 20 & 07 & 3838 & 437 & $\$ 0.08$ \\
2 & 47 & 30 & 6590 & 545 & $\$ 0.13$ \\
3 & 57 & 32 & 9166 & 712 & $\$ 0.18$ \\
\hline
\end{tabular}

Note: Cost calculation based on a charge of $\mathrm{A} \$ 0.02$ per kilobyte

Table 6 Application Use - Server Perspective (mean values shown for simulated access by five simultaneous users)

Average User Wait Time of all Users (ms) Average User Bandwidth ( $\mathrm{kB} / \mathrm{s})$

Hit Count

Hits per second

Average User Wait Time of all URLs (ms)

Total bytes
1428.6

0.5176

5

1.9956

1428.6

2602.8

Table 5 shows the results of the time taken and data quantities sent and received by a user obtaining and reading through the display screens as presented in Figure 10. The data quantities enabled the calculation of an approximate service cost to the user for obtaining the route information.

LBS services are rarely going to be used by a single user, therefore multiple users were simulated accessing the system concurrently. The mean values (for five simulations of five users) of attributes that would impact on an end user's experience of the system were recorded and are shown in Table 6.

\section{Observations and Discussion}

Both the construction and testing of the prototype revealed several issues for each of the SDI requirements related to the topics identified in the observation matrix shown in Figure 9. The construction of the matrix involved hypothesising about the issues of data, standards, policy and access from the perspective of different user groups. Three groups representing end users, integrators and data providers were selected in light of the typical location based service development environment. Issues from the observation matrix are discussed in turn for each of the people categories.

\section{Data Provider - Data}

The role of data providers is to capture data and provide it in an appropriate form for its intended use. Both public and private industry are involved in the provision of various 
data sets for many purposes. Specifically for this version of the prototype, the Victorian Government Department of Infrastructure (DOI) was the sole data provider, contributing data sets representing the spatial relationships between tram stops and tracks. The data was provided in industry format files using a standard projection system facilitating integration with other data sets. The data representation, however, lacked the topology required for network modelling and routing. Provided without metadata, it was difficult to ascertain how the data was originally captured. However DOI did advise that the stop positions had been updated using GPS techniques, the last updates being completed in June 2002. The lack of metadata also hindered assessment of each of the data quality elements. The positional accuracy of stop positions appeared to be planimetrically accurate to within $10 \mathrm{~m}$ (based on data overlays and GPS positioning of several stops). The logical consistency, which assumes consistent measurements will yield similar values when repeated many times, proved to be quite poor with individual lines (that varied in their position) used to represent each tram route despite them being physically coincident. As with many data sets, typographical errors and missing entries for a small proportion of attributes were evident.

\section{Data Provider - Standards}

Since the data was provided using industry standard file formats and projections, integration with other data sets and software systems was easily achieved. The lack of data quality statements and metadata implied that no standards for these items had been adopted.

\section{Integrator - Data}

From the perspective of a data integrator, responsible for combining data sets and developing applications and interfaces to facilitate access and use by end users, several issues emerged. Whilst the data from the data provider was in an industry standard format, it required conversion to an alternative format and restructuring for the purposes of network traversal. These modifications could have an impact on data maintenance policies; if DOI provide updated data on a regular basis this procedure has to be applied for each updated data set.

Developing applications that provide timely responses to requests is also an issue for integrators. To achieve this, software capable of manipulating and querying spatial data based on user's requests was required. Based on studies of attention thresholds and the fixed Internet, pages should load within ten seconds (Nielsen 2000). Given the environmental demands on a mobile user, this limit is probably too generous. Finding the balance between time to input and receive information is essential.

The prototype construction highlighted the inadequacy of 'spaghetti' data sets for topological modelling. For integrators combining data sets and developing navigational location based services, much work may be involved in the preparation of topology for networking purposes. Additionally the management of large data sets, and their subsequent segmentation and restructuring to present meaningful information to mobile users requires significant effort. While this has only been touched upon in this prototype, it is not an issue to be overlooked and will be investigated in more detail as part of this research. 


\section{Integrator - Standards}

The public transport application required standard interfaces to link the various software components; the back-end algorithm implementations needed to be compatible with the Internet and the mobile devices on which the application could be accessed.

Data quality was directly dependent on the data provider. Alterations that were made to the data set were for networking purposes and aimed to enhance the logical consistency of the data sets. Since no metadata records existed, no particular metadata standard was preferred over another. Metadata records were established for the modified data sets using ANZLIC's metadata guidelines (compatible with ISO/DIS 19115) designed for the Australian Spatial Data Directory.

The data model currently incorporates all tram stops within the Melbourne metropolitan region. Based on the work of Huang and Peng (2001), the network need only contain the stops at which interchanges can be made (other stops are redundant). Implementing this would require dynamically constructing the network topology, however with a potential reduction of the number of stops by several thousand this could be a feasible alternative and minimise the problems associated with maintenance updates since less pre-processing would be required.

\section{Integrator - Policy}

Falling within the responsibility of the integrator are policy issues related to privacy and personalisation. With the ability to tailor services to a user's location, and the time of day, this capability enables a very detailed 'digital personae' to be constructed whereby it is possible to determine the nature of a person's activities through the analysis of time (including frequency and duration). It is then possible to identify common signatures of spatio-temporal behaviour and link this to socio-economic data. However, issues of personalisation are also critical to the adoption of such services in terms of cognitive ergonomics (Carroll et al. 1991), and interaction design. This first prototype version did not offer any personalisation features nor impact on user privacy (apart from the wireless network's existing ability to track mobile phones); however, subsequent versions will investigate these issues in depth.

\section{Integrator - Access}

As identified earlier, human computer interaction within the mobile environment is an important issue. The first levels of the proposed hierarchical information structure were implemented (shown in Figure 10) in light of these constraints. In addition to presenting minimal, but sufficient, information to a user conducting various tasks, the response time of the application and its ability to handle multiple simultaneous requests are also of significance. As explained above briefly, in 1968 Miller first proposed that a user's attention to a computer related task can be maintained with delays of up to approximately ten seconds. This attention threshold is still maintained by Web usability guidelines today (Nielsen 2000). Although largely dependent on network speed, mobile users should receive a response from the system within this ten second threshold. The simulated 'real use' testing of the application revealed that the response time for each page 
was 10 seconds on average (refer to Table 4 for details), verifying that each WML page was appropriately sized. As shown in Table 6, even with five simultaneous users performing route requests, the average wait time for all users was just over one second - a minimal delay, and one that would be only just noticeable by a user.

\section{End User - Data}

The nature of mobility implies specific characteristics in terms of data content that is delivered to users. Mobile users do not want to be overloaded cognitively, rather they need relevant and timely information that is easy to read and comprehend. Users appreciate data that is highly accurate and reliable, reducing the cognitive load associated with evaluating and interpreting it. As mentioned previously, input items need to be clear, allow for easy input and not require onerous activity in relation to the resulting information that is displayed.

\section{End User - Policy}

The issues of personalisation and privacy are again of relevance to end users. However, in this version of the prototype the position of the user is not tracked or recorded, and the application has no 'memory' for frequently recorded routes, nor the ability to store frequently visited locations. In future versions where street intersections, rather than stop numbers, will be used to identify location these issues will be explored.

\section{End User - Access}

As discussed previously, issues from an end user's perspective are related to interaction with the service in competition with other tasks. Users do not want their service interaction to be time consuming nor impeded by other concurrent users. The testing revealed that even for a relatively complex route, relevant information could be accessed and read in less than one minute, and for a cost of only eighteen cents (Table 5). Although the user time experiments were subjective, based on approximations of input speed and approximation of a user reading through the information presented, they did suggest that the volume of data presented was appropriate. The hierarchical structuring of information (without thorough assessment by a user group) proved to be a technically feasible approach to conveying varying levels of content without dramatically increasing service response time. Even though GPRS is a packet switched technology and the associated charging policies are based on the amount of data sent and received, the user time and in particular perceived user time is still an important consideration in the usability of these services.

\section{Further Testing}

It should be noted that the initial implementation on which the results and observations are based did not incorporate any timetable information for the trams along the routes. The 'best' tram to catch was based solely on the first route that passed through the 
greatest number of stops. This method of route selection will continue to form the basis in later versions that will include tram timing information, as the best tram to catch may not necessarily be the first tram that passes through the stop. The incorporation of timing information will also enable journey time calculations to be included with the journey itinerary.

Subsequent versions will also provide the option to automatically determine the user's position (assumed to be the journey origin) and prompt only for a destination street address (rather than tram stop number). This will require appropriate pedestrian descriptions from the journey origin to the first tram stop, and similarly at the end of the journey from the last tram stop to the destination. Additionally, the provision for alternate paths that the user could evaluate and choose between could also be provided in future versions.

The testing methodology employed for this prototype version proved useful in examining the observation criteria identified in Figure 9. However, more extensive testing will be performed for subsequent versions of the prototype. Later prototype versions will provide more detailed information, including graphical maps to assist users in planning and executing their journeys. As a result a wider range of sample routes will be tested and the associated time and data quantities recorded. It is also proposed that a range of people representing the categories of end user, data provider and data integrator will be asked to evaluate the system and the nominated observation issues. The evaluation will take the form of trialling the system and completing a questionnaire detailing computing and technology experience, spatial training, feedback about the system, and the underlying infrastructure required to deliver the application.

\section{Conclusions}

Spatial Data Infrastructures provide an effective framework in which to explore the set of interrelated issues that collectively govern the quality of service delivery in LBS. Although data access and dissemination through SDI has previously been considered with a focus on wire line communication methods, the basis of the theoretical principles remains constant when moving to the wireless environment. People still require access to data sets, and the access is achieved through the establishment of appropriate networks, standards and policies. It is the specifications for these components that require modification to accommodate the successful delivery of location based services.

The phased prototype approach has proved, even at the early stage of the first version implementation, to be a useful means by which to examine the SDI components in relation to a location based service in greater detail. While the approach is quite specific, it is anticipated that it will be possible to generalise the principles from all prototype versions and hence define guidelines in order for SDI to support location based services.

The most poignant observations made in the initial phase of this research relate to data and the privacy of the individual. With wireless communication and information access methods expected to continue to evolve and play an increasing role in society, it is critical to determine an infrastructure that meets the needs of this new user base. There exists a real tension between a user's preference for 'spatial and temporal anonymity' and delivery of service whose quality is very much linked to precisely knowing when and where they are. Perhaps users will prefer to make themselves 'selectively available' to different services. 
Whilst SDI can support delivery of services such as the one proposed in this paper, there are important refinements requiring further consideration. These include:

- the criticality of information (assessing impact of real-time information on current user plans);

- time/space indexing (indexing data for fast retrieval and delivery of 'user centred' data);

- data conflation (integration of fine scale, time critical, ephemeral, spatial and aspatial data);

- data formats (support of integration and rendering of information in a range of formats (text, sound, maps, 3D, spoken instruction, photographs, film sequence)); and

- customisation (ease of tailoring interaction and presentation of information).

These refinements will take time, and may very well be part of a continual evolution, particularly as technology continues to adapt and advance. However, they cannot be overlooked if SDI is going to fully support the wireless dissemination of information.

In the short term, SDI practitioners could facilitate the rollout of LBS applications by broadening the perspective of their current initiatives and planning for a future in which reliable, higher bandwidth wireless Internet connections are available for a reasonable price. Maybe a particular data set within an existing SDI would be of benefit to an LBS application; perhaps providing real-time attributes for this data set would be extremely valuable to a mobile end user. Like many other software applications it can be a challenge to get an LBS up and running. Sourcing and gaining access to relevant data sets being one such challenge. If LBS developers could leverage off some of the partnership and data access arrangements already established for various SDI initiatives, the battle would be half won. Adapting the SDI model so that it can support wireless spatial information dissemination is the first step on a path to improving access to spatial information.

\section{Acknowledgements}

Warm thanks are extended to the Carnegie Trust for funds for Dr. William Mackaness to travel and undertake research in Location Based Services at the University of Melbourne in the Department of Geomatics, and to The Department of Infrastructure and Webraska Mobile Technologies (Australia) for their support in the development of this prototype. The authors also wish to gratefully acknowledge the support of Land Victoria and the members of the Centre for Spatial Data Infrastructures and Land Administration at the Department of Geomatics (http://www.geom.unimelb.edu.au/ research/SDI_research/), University of Melbourne in the preparation of this paper and the associated research. The views expressed in the paper are those of the authors and do not necessarily reflect the views of these groups. 


\section{References}

Blonz T and McCarthy C 1998 Mobile Location Services. Boston, MA, Ovum

Bourrie S R 2000 A Sense of Place: Getting There from Here. WWW document, http:// www.wirelessweek.com/news/wirelessip/wip515f.htm

Carroll J M, Kellogg W A, and Rosson M B 1991 The task-artifact cycle. In Carroll J M (ed) Designing Interaction: Psychology at the Human-Computer Interface. Melbourne, Cambridge University Press: 74-102

Chan T O and Williamson I P 1999 Spatial Data Infrastructure management: Lessons from corporate GIS development. In Proceedings of AURISA 99: The 27th Annual Conference of AURISA, 22-26 November, Blue Mountains, NSW (available at http://www.geom. unimelb.edu.au/research/publications/IPW/chan.pdf)

Coleman D J and McLaughlin J 1998 Defining Global Geospatial Data Infrastructure (GGDI): components, stakeholders and interfaces. Geomatica 52: 129-43

Costelloe D, Mooney P, and Winstanley A 2000 Multi-objective optimisation and dynamic routing algorithms in transportation networks. In Proceedings of GIScience 2000, 28-31 October, Savannah, Georgia, USA

Cox D C 1996 Wireless personal communications: A perspective. In Gibson J D (ed) The Mobile Communications Handbook. New York, IEEE Press: 209-41

Dennis M 2001 Location, Location, Location. WWW document, http://www.geojava.com/ company/Michael_Dennis/LocationTechnologyArticle.html

Ericsson 2000 Annual Report 2000. WWW document, http://www.ericsson.com/annual_report/ 2000/eng/index_fl.shtml

Ericsson 2001 Annual Report 2001. WWW document, http://www.ericsson.com/annual_report/ 2001/eng/br/market.shtml

FCC 1999 FCC acts to promote competition and public safety in enhanced wireless 911 services. WWW document, http://www.fcc.gov/Bureaus/Wireless/News_Releases/1999/nrw19040.html

Feeney M-E and Williamson I P 2000 Researching frameworks for evolving Spatial Data Infrastructure. In Proceedings of SIRC 2000: The 12th Annual Colloquium of the Spatial Information Research Centre, University of Otago, 10-13 December, Dunedin, New Zealand (available at http://www.geom.unimelb.edu.au/research/publications/IPW/4_00Feeney.pdf)

Henderson A 1991 A development perspective on interface, design, and theory. In Carroll J M (ed) Designing Interaction: Psychology at the Human-Computer Interface. Cambridge, Cambridge University Press: 254-68

Hoogenraad B 2000 Location dependent services. In Proceedings of the Third AGILE Conference on Geographic Information Science, 25-27 May, Helsinki/Espoo, Finland

Huang R and Peng Z-R 2001 Object-oriented data modeling and trip planning algorithms for dynamic transit networks. In Proceedings of University Consortium for Geographic Information Science Summer Assembly, 20-24 June, Buffalo (available at http://www.cobblestoneconcepts. com/ucgis2summer/huang/huang.htm)

Huisman O and Forer P 1998 Computational agents and urban life spaces: A preliminary realisation of the time-geography of student lifestyles. In Proceedings of the Third International Conference on GeoComputation, 17-19 September, University of Bristol, United Kingdom

Jacobson I, Christerson M, Jonsson P, and Övergaard G 1992 Object-Oriented Software Engineering: A Use Case Driven Approach. New York, Addison-Wesley

Kjeldskov J, Howard S, Murphy J, Carroll J, Vetere F, and Graham C 2003 Designing TramMate: A context aware mobile system supporting use of public transportation. In Proceedings of Designing for User Experiences, 5-7 June, 2003, San Francisco, California, USA

Koh J and Kim Y-M 2000 Mobile Internet Applications Primer. Paris, UBS Warburg

McCabe E 1999 Location-based services offer a global opportunity for new revenue. WWW document, http://www.telecoms-mag.com/default.asp?journalid=3\&func=articles\&page $=$ location \&year $=1999 \&$ month $=10 \&$ srchexpr $=$ McCabe

Molich R and Nielsen J 1990 Improving a human-computer dialogue. Communications of the ACM 33: 338-48

Nielsen J 1994 Enhancing the explanatory power of usability heuristics. In Proceedings of Human Factors in Computing Systems: 'Celebrating Interdependence', 24-28 April, Boston, Massachusetts: $152-58$ 
Nielsen J 2000 Designing Web Usability. Indianapolis, IN, New Riders

Parthus Technologies 2001 Parthus launches and licenses NavStream 3000 GPS platform enabling location determination in the most challenging of indoor and urban canyon environments. WWW document, http://www.parthus.com/news/press_releases/october_09_2001.html

Payne J W, Bettman J R, and Johnson E J 1993 The Adaptive Decision Maker. Cambridge, Cambridge University Press

Peng Z-R and Huang R 2000 Design and development of interactive trip planning for web-based transit information systems. Transportation Research Part C 8: 409-25

Pratt A R 1999 Combinging GPS and cell phone handsets: The intelligent approach. In Proceedings of ION GPS’99, 14-17 September, Nashville, Tennessee: 529-35

Quirion C A 2001 Wireless mapping and navigation: Utilisation and delivery of spatially-enabled content. In Proceedings of SatNav 2001: The Fifth International Symposium on Satellite Navigation Technology and Applications, 24-27 July, Canberra

Rajabifard A, Chan T O, and Williamson I P 1999 The nature of regional Spatial Data Infrastructures. In Proceedings of AURISA 99, Blue Mountains, NSW (available at http://www.geom. unimelb.edu.au/research/publications/IPW/ipw_paper32.pdf.pdf

Rajabifard A, Williamson I P, Holland P, and Johnstone G 2000 From local to global SDI initatives: A pyramid of building blocks. In Proceedings of Fourth Global Spatial Data Infrastructure Conference, 13-15 March, 2000, Cape Town, South Africa (available at http://www.geom. unimelb.edu.au/research/publications/IPW/ipw_paper41.pdf)

Robinson T 2000 Wireless Marketing is about Location, Location, Location. WWW document, http://www.techweb.com/wire/story/TWB20000913S0014

Sacher H, and Loudon G 2002 Uncovering the new wireless interaction paradigm. Interactions 9(1): $17-24$

Singleton L A 1983 Telecommunications in the Information Age: A Nontechnical Primer on the New Technologies. Cambridge, MA, Ballinger

Solymar L 1999 Getting the Message: A History of Communications. Oxford, Oxford University Press

Souissi S and Phillips G 2000 WAP Based Location Services. WWW document, http://www.w3.org/ Mobile/posdep/MotorolaW3C.html 


\section{University Library}

\section{- M M N E R VA A gateway to Melbourne's research publications}

Minerva Access is the Institutional Repository of The University of Melbourne

Author/s:

SMITH, JESSICA;Mackaness, William;KEALY, ALLISON;Williamson, Ian P.

Title:

Spatial Data Infrastructure requirements for mobile location based journey planning

Date:

2003-12

Citation:

Smith, J., Mackaness, W., Kealy, A., \& Williamson, I. P. (2003). Spatial Data Infrastructure requirements for mobile location based journey planning. Transactions in GIS, 8(1), 23-44.

Publication Status:

Published

Persistent Link:

http://hdl.handle.net/11343/33964 